\title{
SALUD, ESTRUCTURA FAMILIAR Y PAUTAS DE DESIGUALDAD: EL CASO DE LAS CONSULTAS PRIVADAS DE SALUD DENTAL
}

\author{
Mauro F. Guillén*
}

Universidad de Yale

RESUMEN. Las teorías de la estratificación social que sirven para conceptualizar el acceso de las personas a los puestos de trabajo y sus retribuciones salariales han empleado siempre la familia como unidad básica de análisis. En el presente trabajo se demuestra la necesidad de abordar el estudio del consumo de bienes o el acceso a servicios por parte de las personas, teniendo en cuenta las desigualdades entre los miembros de la familia. Los diversos miem. bros de la familia, en virtud de su distinta posición estructural, disfrutan de oportunidades de consumo desiguales. A partir de ciertas ideas de Weber y del modelo de elección racional de Hechter, se propone una teoría de dependencia y control entre los miembros de la familia que permite evaluar, empíricamente, las pautas de desigualdad. Para tal fin se analiza la información sobre el acceso a consultas dentales privadas contenida en la Encuesta Nacional de Salud española de 1987. La metodología empleada consiste en modelos de regresión logística, abordándose los problemas causados por la existencia de sesgos de selección muestral, y presentándose ciertas sugerencias para una política de salud dental.

* Una versión anterior de este artículo obtuvo una mención especial del jurado del Concurso Internacional para Jóvenes Sociólogos organizado por la Asociación Internacional de Sociología con ocasión de su Congreso en Madrid en 1990. Debo al profesor Robert Jenkins gran número de indicaciones técnicas y sugerencias teóricas. Agradezco también los comentarios de Scott Boorman, Jesús M. de Miguel, Juan Díez Medrano, Paul DiMaggio, Kai Erikson, José María García Blanco, Rodolfo Gutiérrez; Charles Perrow, Josep Rodríguez, Dimitrios Sotiropoulos y David Williams. El análisis de los datos se realizó en la Universidad de Yale gracias a una beca del Programa de Ayudas a la Investigación del Centro de Investigaciones Sociológicas en 1988. 


\section{ESTRUCTURA FAMILIAR Y SALUD}

La sociología de la estratificación social ha prestado relativamente poca atención a los procesos de desigualdad en el seno de las familias en cuanto a determinantes de la distribución de la renta y del consumo. El campo de la estratificación social ha estado primordialmente interesado en el análisis de las recompensas (renta, estatus) que se derivan de ciertas posiciones en el sistema social, así como en la manera en que las personas consiguen acceder a esas posiciones. Como apuntan Kalleberg y Sørensen (1979: 362), "la bibliografía sociológica ha tendido a concentrar su atención en el estatus ocupativo como la recompensa esencial del trabajo y a recalcar el papel de la educación en la transmisión del estatus familiar a la siguiente generación". Incluso los sociólogos europeos más críticos de la tradición americana de estudios de estratificación social - el modelo de la consecución de estatus social - han afirmado que las desigualdades derivadas del sistema de clases se refieren a dos procesos sociales distintos, a saber, "la asignación de recompensas según las posiciones en el sistema social" y "el proceso de reclutamiento a esas posiciones" (Parkin, 1978: 13).

Típicamente, la familia ha sido la unidad básica de análisis (Acker, 1973; Allen, 1982), sobre todo porque tanto los sociólogos empíricos americanos como los sociólogos marxistas han preferido abordar simultáneamente los procesos de recompensa y reclutamiento. Ambas tradiciones han relegado el consumo o la distribución real de la riqueza y los recursos familiares a un segundo plano. La consecuencia natural ha consistido en el olvido de las características individuales en favor de las características familiares, porque se ha conceptualizado a la familia como el indicador de la clase social de la persona. El reclutamiento a las posiciones sociales también se ha estudiado teniendo en cuenta las características familiares, siendo la educación la variable interviniente. Por ejemplo, Blau y Duncan (1967) y Parkin (1971) consideran que la estructura ocupativa constituye la "columna vertebral" de la estratificación social (Parkin, 1971: 18-19; Wright, 1985), si bien Blau y Duncan emplean en uno de los últimos capítulos de su libro algunas variables intrafamiliares (1967: 295-330). Parkin incluso llega a afirmar que las pautas intrafamiliares de estratificación no son importantes: «la asignación de recompensas sociales y económicas de la gran mayoría de las mujeres aparece determinada primordialmente por la posición social de sus familias $-y$, en particular, por el cabeza de familia, que es un varón" (Parkin, 1971: 14-15). El interés por analizar la reproducción de clase o el sistema de estratificación ha impedido a la mayoría de las investigaciones considerar las pautas de desigualdad que se dan en el seno de las familias (Parkin, 1978: 627), y, en especial, la problemática de las desigualdades en el consumo.

Antes de examinar los factores que se encuentran detrás de la desigualdad y la segregación de roles entre los miembros de la familia, es importan- 
te situar los procesos intrafamiliares dentro del marco más amplio de las pautas interfamiliares de estratificación en la sociedad. Con esto queremos decir que es necesario discutir primero el tamaño del pastel a repartir, el conjunto total de recursos familiares que viene dado por la situación de la familia en el sistema de clases.

La aproximación multidimensional o causal al estudio de las clases sociales ha sido severamente criticada por tender a borrar las líneas divisorias entre las clases sociales con la falacia de un continuo de estratos, y por ser exageradamente simplificadora (Parkin, 1971: 17; 1978: 602). Esta crítica parece más aplicable a estudios sobre sociedades europeas que a los Estados Unidos, porque las líneas de separación tienden a ser más claras y estables en el caso de las sociedades europeas, y la movilidad social ascendente no es tan frecuente como en los Estados Unidos. (Véase, en cambio, el estudio de Lipset y Bendix [1959].) Sin embargo, resulta útil poder situar a las familias según sus recursos de renta y estatus, especialmente cuando el objeto de la investigación son las posibilidades de consumo del individuo. Así, es conveniente pensar acerca de la situación de clase como una situación de mercado, es decir, como una base para la acción social, tal y como indica Weber ([1922] 1978: 926-932). En este marco conceptual weberiano, la afirmación de que "la probabilidad típica de procurarse bienes, alcanzar una posición en la vida y encontrar satisfacciones intrínsecas" constituye el significado práctico de la situación de clase (Weber [1922], 1978: 302) facilita la concepción de la asignación de recursos como un proceso en dos pasos: primero, la determinación del conjunto total de recursos familiares, y, después, la distribución del mismo entre los miembros de la familia.

Parece razonable proponer la renta familiar total como uno de los indicadores de clase o, con mayor exactitud, como un indicador del montante total de recursos disponible para el consumo de los miembros de la familia. Existen problemas, sin embargo, con la variable de estatus social, que nos ayuda a hilar más fino en el posicionamiento de las familias dentro de la estructura social. Como apunta Parkin (1971: 32), si el estatus familiar se mide mediante el prestigio ocupativo del cabeza de familia puede interpretarse como una ventaja social, tal y como argumenta Weber ([1922] 1978: $302-307,937)$. Pero también resulta posible concebir el estatus social como la propensión hacia pautas de consumo aparente (Veblen [1899]), posibilidad que también entrevé Weber en su discusión sobre los estilos de vida y su relación con los grupos de estatus (Weber [1922], 1978: 305-307, 932933).

Una vez conceptualizados los recursos familiares totales, la pregunta de cómo se distribuyen los recursos entre los miembros de la familia resulta plenamente relevante. Uno de los argumentos centrales de esta investigación es que los estudios de estratificación social sobre los procesos individuales de consumo deben tener en cuenta no solamente los que los sociólo. 
gos denominan la posición de la persona en el sistema de clases (determinada por la pertenencia familiar), sino también la posición que ocupa la persona en el seno de la familia. En particular, dada la posición de la familia en el sistema de clases, la distribución intrafamiliar de la educación, la comida, el vestido, el tiempo de ocio y los cuidados sanitarios puede ser tremendamente desigual. Por tanto, los procesos intrafamiliares de toma de decisiones y la dinámica de poder, así como la aparición de conductas egocéntricas o heterocéntricas, se convierten en temas de investigación relevantes. Como apunta Michael Young en un estudio clásico de desigualdad intrafamiliar (1925: 305), "se ha asumido con frecuencia, si bien no de modo explícito, que la familia, aunque ya no se la puede considerar como una unidad en cuanto a su capacidad de generar rentas, puede todavía considerarse como una unidad de gastom. (Véanse, también, Galbraith, 1973: 2937; Delphy, 1979.) Existen numerosos estudios sociológicos sobre la pobreza y el desempleo en Gran Bretaña entre 1850 y 1950 que atestiguan la invalidez del supuesto de que la posición intrafamiliar de la persona es irrelevante a la hora de evaluar su nivel de vida (Oren, 1973; Young, 1952). Merece la pena citar otras revisiones críticas de ciertas políticas públicas en el campo del bienestar social que denuncian que un supuesto implícito de las políticas sociales sobre la familia ha sido la idea de que el dinero llega al hogar para ser repartido equitativamente entre los miembros del mismo" (Pahl, 1980: 315; véase, también, Kincaid, 1979: 217).

No fue hasta finales de la década de los años sesenta que la sociología de la estratificación social comenzó a prestar una mayor atención a las características individuales, especialmente a las distinciones raciales o étnicas y las existentes entre mujeres y varones (Parkin, 1978). Las características de la persona resultaron ser muy importantes a la hora de explicar las desigualdades en el mercado de trabajo, tanto en los Estados Unidos como en Europa (Kelsall, Kelsall y Chisholm [1974]: 134-145). Incluso algunos sociólogos marxistas admitieron la existencia de una desigualdad social considerable que aparece "ostensiblemente independiente de la clase social", mencionando que la segregación por sexo es quizá el componente más importante (Westergaard y Resler, 1975: 97). Otros estudios marxistas recientes argumentan, sin embargo, que uen las sociedades capitalistas, incluso a pesar del carácter partriarcal de las relaciones en el seno de las familias, la unidad primaria de consumo es la familia" (Wright, 1985: 225). En general, las características personales se conceptualizan como factores que acentúan o alivian las desigualdades de clase. Westergaard y Resler avanzan la idea de que "el efecto [de las desigualdades entre mujeres y varones] podría ser el de una alteración de las desigualdades de clase, y quizá podría limar sus vértices más sobresalientes» (1975: 97). Estudios recientes dentro de la tradición de la consecución de estatus social han empleado datos norteamericanos, y de países de Europa Occidental y Oriental, demostrando que la discriminación racial y sexual en el mercado de trabajo 
no se explica completamente mediante las diferencias existentes en cuanto a la procedencia familiar, la experiencia de trabajo, la educación o la productividad de los distintos grupos raciales y sexuales (Bielby y Baron, 1986; Blossfield, 1987; Jenkins, 1987; Kalleberg y Sørensen, 1979; Treiman y Ross, 1983).

La relevancia de las pautas intrafamiliares de estratificación no debe ser exagerada cuando el objeto de la investigación son problemas no relacionados con el consumo. Por ejemplo, la discriminación sexual en las sociedades modernas parece ser el producto de pautas distintivas de organización burocrática del trabajo que meramente el resultado de procesos intrafamiliares anteriores que hayan podido lastrar a algunas personas y a otras no (Baron, 1984; Kanter, 1977).

El contexto general en el que enmarcamos la discusión teórica y empírica sobre los procesos de estratificación en el seno de las familias se define mediante la proposición de que a lo largo de las últimas tres décadas el modelo de familia patriarcal, típico de las sociedades occidertales, ha dado paso a otro modelo más igualitario de familia nuclear que parece una asociación o grupo de miembros a los que se les permite participar en el proceso de toma de decisiones sobre la distribución de recursos. Los estudios sobre la estructura de poder familiar y los procesos de toma de decisiones se multiplicaron en Gran Bretaña y los Estados Unidos, aunque la mayoría de ellos se centraban en el análisis de las diferencias de poder entre marido y mujer sin tener en cuenta a las otras personas dependientes que viven en el hogar (Safilios-Rothschild, 1970; Gillespie, 1971; Edgell, 1980: 53-71). Aunque los resultados contenidos en estos estudios no resultan comparables entre sí, pueden resumirse diciendo que las desigualdades intrafamiliares de poder y los procesos de desigualdad en el consumo de recursos son aún la norma (Gillespie, 1971).

El marco teórico que proponemos consiste en evaluar la posición de los miembros de la familia por medio de los resultados de los procesos de toma de decisiones en el seno de las familias. En estos procesos de toma de decisiones se distribuyen recursos tales como la renta, la situación de clase y el prestigio o estatus (Safilios-Rothschild, 1970: 540). La manifestación más importante de estas decisiones sobre la distribución de recursos son los diferentes niveles de consumo de cada miembro familiar. Resulta útil concebir los procesos de distribución familiar como procesos en dos pasos, siguiendo ciertas teorías económicas sobre la familia (Willis, 1987: 73). En. primer lugar, las familias maximizan la riqueza familiar total o, en términos sociológicos, la posición de la familia en el sistema de clases sociales. Asumiremos que esta posición de la familia viene dada, dejando a un lado, por tanto, el modo en que la distribución interna de recursos puede afectar a la riqueza familiar total, asunto que se considera de especial relevancia en los estudios sobre el subdesarrollo (Rosenzweig, 1986). En segundo lugar, los recursos familiares totales dentro de cada período de tiempo considera- 
do se encuentran disponibles para ser distribuidos entre los miembros de la familia. El problema consiste en establecer las reglas para distribuir los recursos. La teoría económica argumentaría que la distribución intrafamiliar de recursos procedería según una suma ponderada de las utilidades de cada persona, siendo las ponderaciones una medida del grado de altruismo (Willis, 1987: 75). En sociología, estamos más interesados en otras variables como la autoridad, influencia o el poder como fuerzas que influyen en las consecuencias de las actuaciones egoístas o heterocéntricas de las personas. Estas consecuencias son las que determinan conjuntamente la probabilidad de que un miembro familiar pueda asegurarse suficientes recursos para acceder a determinados tipos de consumo. Si consideramos la estratificación en el acceso a cuidados sanitarios especializados, como los de salud dental, se puede asumir que, controlando por la necesidad fisiológica, los miembros familiares en posiciones de autoridad, influencia o poder tendrían una mayor capacidad de decisión sobre la distribución de recursos, y, si se comportaran de modo egoísta, sería más probable que ellos fueran los que accedieran a servicios de salud dental caros.

Es preciso formular una teoría sociológica que permita avanzar qué tipo de características individuales o posiciones dentro de la familia tienden a aumentar la autoridad, la influencia o el poder. Las teorías sociológicas de la elección racional pueden contribuir a formular tales teorías. El sociólogo Michael Hechter, en su libro Principios de solidaridad en los grupos (1987), ha aplicado su versión de la elección racional a los procesos de distribución de recursos dentro de las familias. Un supuesto básico del modelo de Hechter consiste en asumir que las personas se comportan como individualistas metodológicos y gozan de capacidad de decisión y elección. La única restricción a su capacidad de obrar y tomar decisiones se refiere a la suma total de los recursos familiares, que viene determinada por la posición de clase de la familia. Hechter (1987: 21) asume, además, que

«el ejemplo más frecuente [de una Gemeinschaft o comunidad] es la relación familiar. Los padres se sienten obligados a tener en cuenta todos los aspectos relacionados con el bienestar de sus hijos e hijas; $y$ el voto matrimonial exhorta a ambos cónyuges a que se respalden el uno al otro en lo bueno y en lo malon.

Esta concepción es similar a la sostenida por Weber en su concepto de Hausgemeinschaft (comunidad del hogar), el tipo más frecuente de grupo económico, en el que se localiza una acción social intensa y continua, y que se caracteriza por la residencia en común y el comunismo consumista (Weber [1922], 1978: I, 356-360). En otras palabras, la familia (nuclear) se presenta como un grupo social en el que tanto la dependencia como el control entre los miembros son elevados.

Hechter (1987: 54) señala que «las perspectivas de que se dé solidaridad 
de grupo serán más halagüeñas en situaciones en las que las personas dispongan de fuentes limitadas de beneficio, carezcan de oportunidades múltiples de afiliación a grupos, y su aislamiento social sea extremom. Esta idea también la anunció Weber ([1922] 1978: I, 375) cuando escribió que

"con la multiplicación de las posibilidades de vida y las oportunidades, la persona individual se contenta cada vez menos con estar limitada a las formas de vida rígidas e indiferenciadas prescritas por el grupo. Cada vez más, deseará configurar su vida como un individuo y disfrutar de los frutos de sus propias capacidades y trabajo tal y como tenga a bien disponer".

En términos de poder o influencia, los miembros de una familia que trabajan fuera del hogar y perciben ingresos son también miembros de otros grupos externos. Es más probable que personas como éstas se aseguren el acceso a determinados bienes o servicios o, cuando menos, puedan ejercer cierta influencia sobre las decisiones de distribución de recursos en el seno de la familia. Como ha apuntado Galbraith (1973: 35), "la percepción de renta [...] lleva aparejada la autoridad básica sobre su empleo". Además, los miembros familiares que ocupan posiciones de autoridad - como es el caso del cabeza de familia - gozan de una probabilidad mayor de acceder a bienes o servicios.

La investigación empírica sobre pautas intrafamiliares de estratificación con datos de, sociedades desarrolladas arroja resultados contradictorios. Existe evidencia empírica amplia y consistente de que las personas en posición de cabeza de familia se reservan por sí un trozo desproporcionado de la tarta total de recursos familiares (Edgell, 1980: 53-71; Young, 1952). La educación parece estar correlacionada con una mayor capacidad de acaparar recursos familiares (Gillespie, 1971: 452). Aumentos en el tamaño familiar, en particular en el número de hijos o hijas, parece tener efectos negativos para las mujeres pero positivos para los varones (Gillespie, 1971: 456). Sin embargo, el efecto de encontrarse empleado fuera del hogar no queda bien perfilado por la investigación empírica disponible. Gillespie afirma que en los Estados Unidos "las mujeres que trabajan tienen más poder frente a sus maridos, con independencia de la raza o la clase social" (Gillespie, 1971: 452). Edgell (1980: 70) confirma esa relación con datos recopilados en Gran Bretaña. Szinovacz (1977), sin embargo, emplea datos sobre mujeres austríacas concluyendo que el empleo de la mujer fuera del hogar no provoca, necesariamente, relaciones más igualitarias entre los cónyuges. El autor se refiere a variables subculturales, situacionales y de apoyo social para entender mejor los casos en que sí se da una nivelación de los roles. Safilios-Rothschild (1970: 547-548), por su parte, cita varios estudios europeos que demuestran que el empleo fuera del hogar, el tipo de ocupación y 
la educación no pueden considerarse como atributos que aumenten el poder de las mujeres o de los varones en el seno del hogar.

No todas las familias se encuentran igualmente expuestas a los efectos igualadores o democratizadores del empleo fuera del hogar por parte de las mujeres o del mayor nivel educativo de alguno de sus miembros. Parecen existir interacciones interesantes entre la posición de clase de la familia y las variables intrafamiliares de estratificación. Algunos estudios han formulado y respaldado empíricamente la hipótesis de que la transición desde el modelo familiar patriarcal hasta el modelo igualitario ha sido más rápida en las familias pertenecientes a las clases altas, con ingresos elevados, o con niveles educativos altos (Young y Willmott, 1973: 65-100). Un resultado general compartido por los estudios de consecución de estatus social y los de corte marxista es que "la desigualdad salarial por sexo [...] refuerza la desigualdad de clase: es más pronunciada en los estratos inferiores de la jerarquía ocupativa" (Westergaard y Resler, 1975: 101). Gillespie (1971: 452) ha demostrado que los maridos con un nivel educativo elevado tienen propensiones menores al consumo propio aun cuando aumentan sus ingresos personales o su estatus social.

En este artículo se analizan directamente las pautas intrafamiliares de estratificación en virtud del modelo teórico construido a partir de las teorías de Hechter (1987) y de Weber ([1922] 1978) sobre la desigualdad en el seno de las familias nucleares. Para ello se analizan los factores causantes de la consulta a dentistas privados en lugar de a dentistas públicos. Se abordan simultáneamente dos problemas empíricos: 1) la posible existencia de sesgo de selección muestral; y 2) la dinámica inter e intrafamiliar en las pautas de estratificación. El sesgo de selección proviene del hecho de que solamente se observa si una persona acude a dentista privado en el caso de que haya acudido alguna vez al dentista. Para las personas que nunca han acudido al dentista no se dispone de información. Conviene, por tanto, "controlar" el efecto de esta exclusión muestral que bien pudiera acarrerar sesgos o tergiversaciones de las relaciones estadísticas que se observan. El problema a investigar es la desigualdad en el acceso a los cuidados de salud dental en España. En concreto, se analizan las causas de que las personas acudan a consulta dental privada en lugar de a consulta pública, a saber, la proximidad a los centros donde se ofrecen servicios más baratos, el nivel de recursos económicos de la familia, y la posición que la persona entrevistada ocupa dentro de la familia.

\section{LAS CONSULTAS DE SALUD DENTAL: PUBLICAS Y PRIVADAS}

Los servicios de salud dental han recibido, hasta ahora, una atención limitada por parte de la sociología de la medicina y de la estratificación 
social. Se trata, sin embargo, de uno de los aspectos de la sanidad que provoca más desigualdades sociales. Un empuje importante a la investigación en este campo se produce con la publicación de los informes International Dental Care Delivery Systems (Oral Health Unit, 1980), International Collaborative Study of Dental Manpower Systems (Ingle y Blair, eds., 1978) y Oral Health Care Systems (Arnljot et al., eds., 1985). Los tres informes fueron respaldados por la Organización Mundial de la Salud y se desarrollaron entre 1972 y 1983 . El resultado más importante de estos informes es que existen diversos tipos de sistemas de sanidad dental, desde los predominantemente públicos hasta los mayoritariamente privados: sistemas "de pago" como los de Australia, Canadá, Irianda y los Estados Unidos; sistemas conectados a fondos de Seguridad Social como los de Alemania Occidental; sistemas mixtos con fondos públicos y seguros privados como el de Japón; sistemas mixtos de fondos públicos y pagos por parte del paciente como los de Nueva Zelanda y Noruega; 'y, por último, sistemas de policlínicas como los de Alemania Oriental-y Polonia (Cohen et al., 1987: 420).

En España, solamente un 30 por 100 de todas las consultas a dentistas tienen lugar en clínicas públicas, mientras el 70 por 100 ocurren en consultas privadas "de pago" o en sociedades médicas privadas. Las diversas organizaciones benéficas atienden a menos del 1 por 100 de las consultas. Esta situación es precisamente la inversa a la que opera en el conjunto del sector sanitario español, de acuerdo con la famosa ley del 70/30 entre los sectores público y privado (De Miguel, 1985, 1986). Podemos concluir, por tanto, que el sistema español de cuidados dentales se asemeja al sistema mixto de servicios públicos y pagos privados de Nueva Zelanda o Noruega (Davis, 1981). Existen otros dos hechos importantes que conviene tener en cuenta. El primero es que el Sistema Nacional de Salud, que en 1987 cubría a un 95 por 100 de la población española, ofrece solamente dos tipos de cuidados dentales: la extracción de piezas y la asistencia en caso de infección bucodental. Para recibir cualquier otro servicio bucodental (empastes, prótesis, endodoncia, profilaxis, revisiones periódicas), la población española tiene que acudir a dentistas privados y ha de pagar por ello, a no ser que disponga de un seguro privado que cubra tales servicios. (Esta posibilidad era bastante rara en 1987.)

El segundo hecho importante a tener en cuenta es que la profesión dental española hasta finales de los años ochenta era parecida a la italiana, es decir, el dentista era un especialista médico. Al igual que ocurrió en Italia hace unos años (Orzack, 1981), España ha abierto nuevas facultades de odontología que son independientes de las facultades de medicina. Esta situación histórica ha significado que el número de dentistas sea reducido. En 1964 existían solamente 92 dentistas por cada millón de habitantes, proporción que ascendió a 104 en 1974, tras diez años de crecimiento económico inusitado. En 1982 la proporción alcanzó solamente los 107 dentistas por cada millón de habitantes, cifra solamente un poco inferior a la 
italiana (130). En cambio, el resto de los países ricos disponían en 1982 de muchos más dentistas por cada millón de habitantes: Francia (586), Estados Unidos (571), Alemania Occidental (547), Canadá (482), Japón (476) y el Reino Unido (320). Incluso países más pobres que España, como Grecia (794), disponían de más dentistas (OCDE, 1985: 91). El dentista medio europeo suele ganar menos que el médico medio, a excepción de aquellos que trabajan en Alemania Occidental (OCDE, 1985: 59-62). Aunque no existen datos comparables sobre el caso español, es bastante improbable que el dentista medio gane menos que el médico medio.

La falta de un sistema de seguros dentales y la escasez relativa de profesionales dentales en España indica que es posible que se den desigualdades sociales importantes en cuanto a salud bucodental, tal y como se ha estudiado en un capítulo anterior. En este capítulo se investigan las desigualdades en el acceso a cuidados privados de salud dental.

\section{DATOS Y METODOS}

Los datos empleados en el análisis empírico proceden de la Encuesta Nacional de Salud, realizada por el Centro de Investigaciones Sociológicas. El trabajo de campo se realizó en julio de 1987. La muestra probabilística ascendió a casi 30.000 mujeres y varones de 16 y más años, con un error de más/menos 0,51 por 100 y un nivel de confianza del 95,5 por 100. Las definiciones y estadísticas descriptivas de las variables a emplear aparecen en la tabla 1. La variable de renta familiar ha sido estimada mediante un modelo de regresión semilogarítmico a partir de las características sociodemográficas del cabeza de familia, dado que se carecía de información para el 40 por 100 de la muestra. La renta se ha convertido en una variable continua a partir de la información en forma de intervalos asignando a cada persona el punto medio del intervalo correspondiente. En el caso del intervalo abierto superior, el valor se determinó ajustando una curva de Pareto a la distribución cumulativa (Shryock et al., 1980: 365-367).

El prestigio ocupativo del cabeza de familia es una variable continua en la que los puntos de prestigio dependen de la ocupación en función de la clasificación realizada por Treiman (1977) en su Escala Internacional Estándar de Prestigio Ocupativo. La asignación de puntos a cada una de las categorías ocupativas contenidas en la Encuesta Nacional de Salud aparece detallada en la tabla 2. Estas dos variables - renta familiar y prestigio ocupativo del cabeza de familia - sirven para medir el nivel de recursos a disposición del conjunto de miembros de la unidad familiar. Otras dos variables sirven de indicadores de la proximidad a cuidados dentales baratos: la urbanización, que es una variable continua medida con el punto medio del intervalo correspondiente de tamaño del núcleo de población, excepto para Madrid y 


\section{TABLA 1}

Definición y estadisticas descriptivas de las variables dependientes e independientes. Muestra de personas de 16 y más años de edad que no viven solas y que han acudido alguna vez al dentista

Privado

Si la persona entrevistada acudió a dentista privado o de una sociedad médica la última vez que fue al dentista.

Renta Renta familiar estimada (miles de pesetas anuales).

Prestigio Puntuación de prestigio ocupativo del cabeza de familia.

Frontera Si la familia reside en las provincias de Gerona, Huesca, Navarra o en las del País Vasco.

Barcelona Si la familia reside en la provincia de Barcelona.

Urbanización Grado de urbanización del núcleo de residencia familiar (población en miles).

Educación Años de educación completados por la persona entrevistada.

Universidad Si la persona entrevistada ha asistido a la universidad.

Trabaja Si la persona entrevistada se encuentra empleada fuera del hogar.

Cabeza Si la persona entrevistada es cabeza de familia.

Miembros Número de miembros de la familia.

Lambda Relación logística de probabilidad midiendo la probabilidad de que la persona entrevistada haya ido alguna vez al dentista.

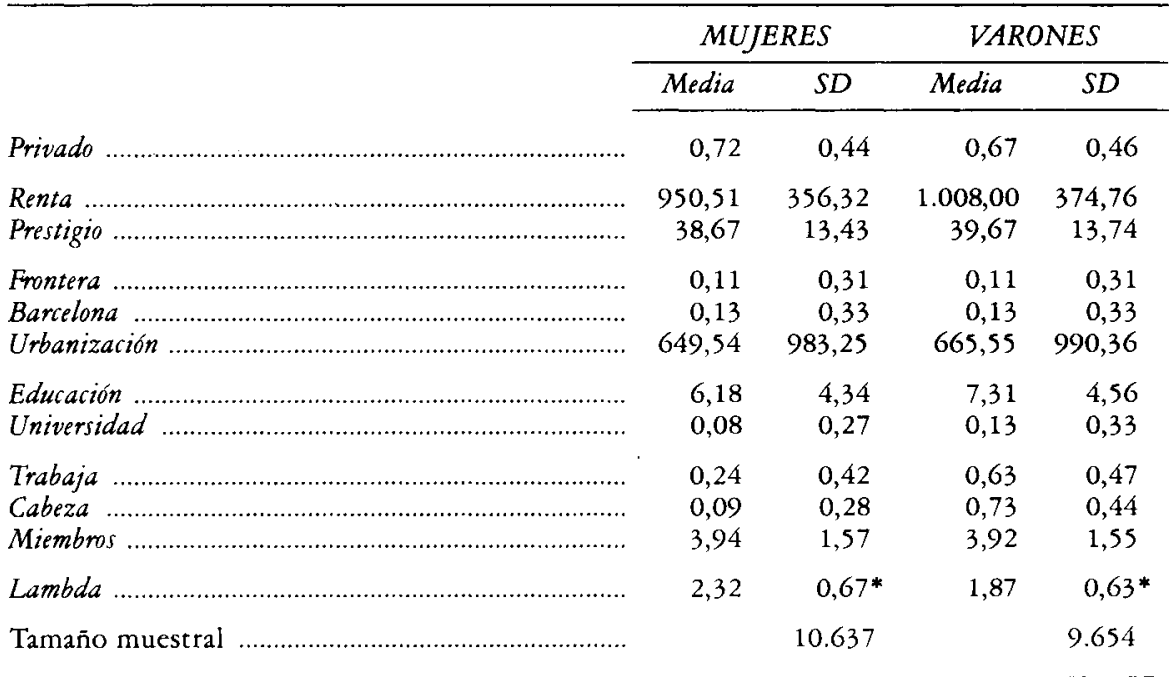

* Los valores medios de lambda corresponden a una probabilidad de 0,91 (mujeres) y 0,87 (varones). 


\section{TABLA 2}

Puntuaciones de prestigio ocupativo según la "Standard International Occupational Prestige Scale" (SIOPS)

\begin{tabular}{|c|c|c|}
\hline Categoria ocupativa & $\begin{array}{l}\text { Código } \\
\text { SIOPS }\end{array}$ & $\begin{array}{l}\text { Puntuacion } \\
\text { de prestigio }\end{array}$ \\
\hline EMPREAGR1: Empresarios agrícolas sin asalariados ......... & 06112 & 38 \\
\hline 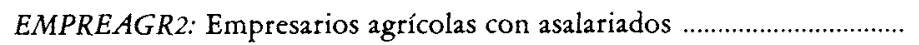 & 06111 & 63 \\
\hline $\begin{array}{l}\text { EMPREIS1: Empresarios industriales y de servicios sin asala- } \\
\text { riados }\end{array}$ & 02190 & 58 \\
\hline $\begin{array}{l}\text { EMPREIS2: Empresarios industriales y de servicios con } \leqslant 5 \text { asala- } \\
\text { riados }\end{array}$ & 02112 & 63 \\
\hline $\begin{array}{l}\text { EMPREIS3: Empresarios industriales y de servicios con }>5 \text { asala- } \\
\text { riados }\end{array}$ & 02111 & 70 \\
\hline 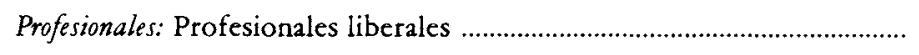 & 00 & 58 \\
\hline 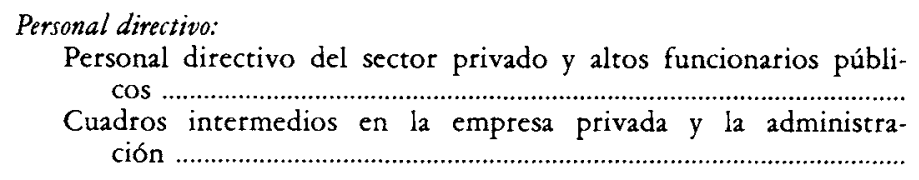 & $0203-11$ & $65,5^{*}$ \\
\hline 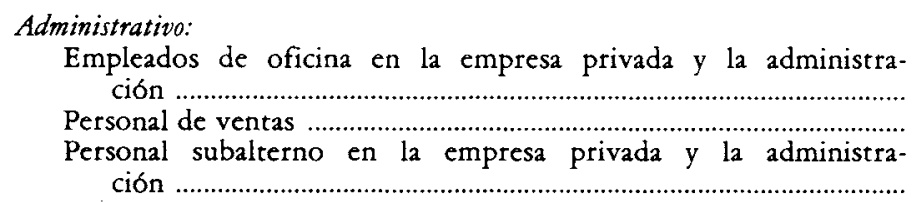 & $\begin{array}{r}03 \\
043 \\
039\end{array}$ & $\begin{array}{l}41 \\
46\end{array}$ \\
\hline 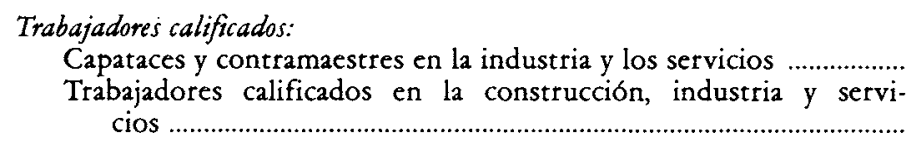 & 09950 & 46 \\
\hline 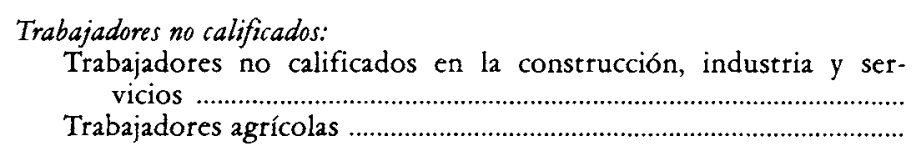 & $\begin{array}{l}0999 \\
0621\end{array}$ & $\begin{array}{l}18 \\
20\end{array}$ \\
\hline 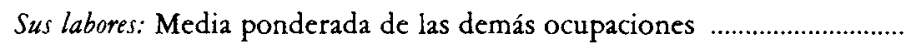 & 一 & 38,73 \\
\hline
\end{tabular}

* La puntuación es la media de las correspondientes a los códigos 0203 y 0211.

FUENTE: Treiman (1977: 235-260). 
Barcelona, en que se introdujo el número respectivo de habitantes; y dos variables dicotómicas, una para las personas que residen en una de las provincias situadas cerca de la frontera francesa (las tres provincias vascas, Navarra, Huesca y Gerona), y otra para las personas residentes en Barcelona, donde los precios de cuidados dentales en las consultas privadas no son tan elevados como en el resto del país. Evidentemente, la proporción de urbanización también mide el posible mayor interés cultural por la salud dental de las personas que residen en núcleos de población de mayor tamaño. Por último, cinco variables sirven para medir la distribución intrafamiliar de recursos. Dos variables dicotómicas sirven para distinguir las personas entrevistadas que trabajan fuera del hogar de las que no lo están, y las que son cabeza de familia de las que no lo son. La educación de la persona entrevistada se mide mediante una variable continua de años de educación formal recibidos, y otra variable dicotómica que distingue las personas que han acudido a la universidad de las que no. Evidentemente, las variables de educación miden también la mayor información de que se dispone acerca de la conveniencia de ir al dentista de modo regular. La quinta variable intrafamiliar es miembros, el número de personas que componen la unidad familiar, que nos sirve para controlar por el número de personas que se reparten el total de recursos familiares.

El análisis se ha verificado mediante modelos de regresión logística (logit), es decir, modelos de regresión modificados para poder ser aplicados al caso de variables dependientes dicotómicas, tal como el acudir a consulta dental privada en lugar de pública. Se trata de una transformación logarítmica de la variable dependiente entendida como una probabilidad; por ejemplo, la probabilidad de acudir a un dentista privado en lugar de acudir a la Seguridad Social. Así, para cada observación y en la muestra obtenemos:

$$
\log _{c}[\mathrm{Pi} /(1-\mathrm{Pi})]=\sum_{\mathrm{j}=1}^{\mathrm{J}} \beta_{\mathrm{ij}} \mathrm{Xij}+\mathrm{Ui}
$$

donde $j=1,2, \ldots, J$ indica las distintas variables independientes, que pueden ser continuas o categóricas. Este modelo tiene varias propiedades que favorecen enormemente su uso en la investigación sociológica (Aldrich y Nelson, 1984; Hanushek y Jackson, 1977: 179-216). Los parámetros estimados $(\beta j)$ han de ser transformados de nuevo para observar el impacto de las variables independientes $(X j)$, si bien en el presente trabajo solamente nos interesa considerar la bondad del ajuste a los datos de distintos modelos jerárquicos (medida mediante un estadístico chi-cuadrada), así como el signo de los parámetros y su significatividad estadística (también evaluada mediante un estadístico chi-cuadrada). El método de estimación empleado 
es una versión modificada de la estimación máximo-verosímil de GaussNewton con pasos compartidos (Harrell, 1986).

Una vez que se eliminaron todas las personas para las que se carecía de alguna información básica (en torno al 6 por 100 de la muestia inicial de 30.000 mujeres y varones de 16 y más años), la muestra de mujeres asciende a 11.988 y la de varones a 11.566 . A todas estas personas se les preguntó si habían acudido alguna vez al dentista en el pasado. Nuestro objetivo consiste en averiguar las pautas de desigualdad en el acceso a dentista priva$d o$, suponiendo que la persona entrevistada ha ido alguna vez al dentista. Pero no todas las personas mayores de 16 años han ido al dentista. El 89 por 100 (10.637) de las mujeres habían acudido al menos una vez, mientras que el porcentaje para los varones es del 84 por 100 (9.654). Si descartamos ese 11 por 100 de mujeres y 16 por 100 de varones por falta de datos sobre la visita a dentista público o privado, incurrimos en lo que se denomina un "sesgo sistemático de selección en la muestra". Esta es una situación muy común en las encuestas sociales. En nuestro caso, el hecho de que proporciones tan importantes de personas no hayan ido nunca al dentista es sospechoso. Evidentemente, una persona puede no necesitar ir al dentista porque su salud bucodental es excelente. Pero es bastante más posible que sean personas muy desaventajadas en términos socioeconómicos las que nunca hayan ido al dentista. Por tanto, si no introducimos algún tipo de control para eliminar el sesgo de autoselección corremos el riesgo de infraestimar la importancia de la discriminación de acceso a los cuidados privados de salud dental. El sesgo de selección se encuentra también presente en los estudios de salarios. Es obvio que si una persona no trabaja no podemos conocer el salario que gana. Obsérvese, sin embargo, que hay muchas mujeres (y algunos varones, sobre todo jóvenes) que no trabajan porque el salario que percibirían sería seguramente muy pequeño. Por tanto, un análisis de regresión a partir de una muestra de salarios para personas que trabajan contiene un sesgo de selección importantísimo. En muchos estudios sociológicos existe la posibilidad inadvertida de encontrar sesgos de selección (Berk, 1983; Huff, 1982: 11-26).

Pero volvamos a nuestro ejemplo. Parece claro de que nos enfrentamos a un problema de sesgo de selección, que, además, es má agudo en el caso de los varones que en el de las mujeres. Heckman (1979) sugiere una manera en que puede neutralizarse el efecto del sesgo de selección. Empleando las muestras totales de 11.988 mujeres y 11.566 varones estimamos la probabilidad de haber acudido al dentista (público o privado) en el pasado. Para ello formulamos un modelo de regresión logística en el que la variable dependiente es igual a cero si no se ha visitado nunca un dentista, e igual a uno en caso contrario. Los modelos logit estimados para mujeres y varones son los siguientes: 
Mujeres: $-1,974+0,000312$ renta $+0,801$ frontera $+0,000164$ urbanización $+0,089$ educación $+0,129$ edad $-0,00107$ edadsq

Varones: $-1,843+0,000300$ renta $+0,005$ prestigio $+0,415$ frontera +

$+0,000134$ urbanización $+0,068$ educación $+0,096$ edad -

- 0,00072 edadsq

donde edad es la edad en años, edadsq es la edad elevada al cuadrado, y el resto de las variables aparecen definidas en la tabla 1 . Todos los parámetros estimados son significativos. Nótese que la ecuación de mujęres no contiene la variable prestigio porque se halló que no era significativa, y, por tanto, se prefirió escoger el modelo en el que esta variable no estaba incluida. Todas las demás variables que no aparecen reseñadas no resultaron significativas y, por tanto, no se tuvieron en cuenta.

Heckman (1979) indica que una vez que se han estimado estas dos ecuaciones se sustituyan en ellas los valores de las variables independientes correspondientes a las 10.637 mujeres y los 9.654 varones para los que sí disponemos de datos sobre consultas dentales. Por tanto, obtenemos un número para cada mujer y cada varón en la muestra, que denominaremos lambda. Por ejemplo, una mujer hipotética que pertenece a una famila con renta anual de 2 millones de pesetas, que no vive en una provincia cerca de la frontera francesa, que reside en un núcleo de población de 10.000 habitantes, que ha estado escolarizada durante doce años, y que tiene 25 años de edad, el valor de lambda es:

$$
\begin{aligned}
\text { lambda }= & -1,974+0,000312(2.000)+0,801(0)+0,000164(10)+ \\
& +0,089(12)+0,129(25)-0,00107(625)=2,28
\end{aligned}
$$

Nótese que los valores de lambda son los de la función logística. Por tanto, la probabilidad de que esta mujer hubiera ido al dentista (público o privado) al menos alguna vez en el pasado es:

Prob (haber acudido al dentista en el pasado) $=\exp ($ lambda) $/[1+\exp$ $($ lambda) $]=\exp (2,28) /[1+\exp (2,28)]=0,9072=90,72$ por 100

En la nota a la tabla 1 se lee que la media de probabilidad de que una mujer en la muestra hubiera acudido al dentista en el pasado es del 91 por 100 (lambda $=2,32$ ). Por tanto, nuestra mujer hipotética es muy parecida a la mujer media de la muestra, solamente un poco menos probable de haber ido al dentista al menos una vez en el pasado.

Ahora que hemos obtenido un valor de lambda (la función logística) o de la probabilidad (puesto que conociendo uno se puede calcular el otro), Heckman (1979) demuestra que si se incluye lambda (o la probabilidad) como variable independiente en todas las regresiones que estimemos con la 
muestra de 10.637 mujeres o la de 9.654 varones, se consigue eliminar el sesgo de selección en la muestra.

Es preciso dejar bien claro en este momento por qué no incluiremos en ninguna regresión la variable edad. Teóricamente, la edad es un factor potencialmente importante: la salud bucodental suele deteriorarse con el paso de los años y las pautas culturales de valoración de la salud son, en gran medida, generacionales. Pero tenemos buenas razones también para pensar que la edad es un factor importante a la hora de ir al dentista en general, pero no a la hora de decidir si se acude a un dentista público o privado. Esta es la razón por la que incluimos la edad en las dos regresiones para obtener el valor de lambda, pero a partir de ahora ya no especificaremos el efecto de la edad separadamente, sino que lo medimos a través del valor de la función logística que representa la probabilidad de haber acudido al dentista al menos una vez en el pasado.

\section{RESULTADOS EMPIRICOS}

Todas las regresiones se han calculado separadamente para mujeres y varones. No solamente aquéllas acuden más al dentista, sino que lo hacen con mayor frecuencia a consultas privadas. El 72 por 100 de las mujeres que han acudido alguna vez al dentista fueron a una consulta privada la última vez, frente al 67 por 100 de los varones. La tabla 3 presenta los valores de los estadísticos chi-cuadradas para varios modelos que incluyen diversos grupos de variables. Este análisis persigue medir la importancia de nuestros tres grupos de variables: nivel de recursos familiares, proximidad a centros de prestación de cuidados dentales, y posición que ocupa la persona entrevistada dentro de la familia. La diferencia entre las chi-cuadradas de dos modelos jerárquicos es también un estadístico distribuido según la ley chi-cuadrada. Por tanto, si esa diferencia es significativa, podemos concluir que las variables adicionales incluidas en el modelo más complejo contribuyen a explicar mejor las causas por las que las personas entrevistadas acudieron a dentista privado la última vez que visitaron uno.

Se observa que la contribución explicativa de las características individuales (educación, universidad) y las que nos indican la posición familiar de la persona entrevistada (cabeza, trabaja, miembros) es significativa. Estos resultados confirman que las desigualdades de acceso individual de las personas a las consultas privadas de salud dental no pueden evaluarse por completo sin tener en cuenta variables de carácter intrafamiliar. Lo más interesante es que, con la única excepción de la adición de la variable de miembros (modelo D frente al modelo C), los incrementos en las chi-cuadradas son mucho mayores en el caso de las mujeres que en el de los varones. Este resultado sugiere que las variables de estratificación intrafamiliar son más 


\section{TABLA 3}

Contribucion de las variables familiares, individuales $e$ intrafamiliares a la explicación de las pautas de acceso a cuidados privados de salud dental

\begin{tabular}{|c|c|c|c|}
\hline \multirow{2}{*}{\multicolumn{2}{|c|}{ Modelos }} & \multicolumn{2}{|c|}{ Chi-cuadrada } \\
\hline & & Mujeres & Varones \\
\hline A) & Renta, prestigio, frontera, Barcelona y urbanización & 575 & 652 \\
\hline B) & 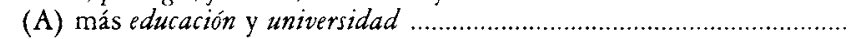 & 724 & 722 \\
\hline C) & 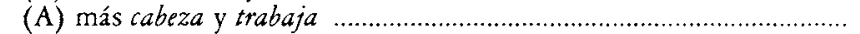 & 654 & 675 \\
\hline D) & 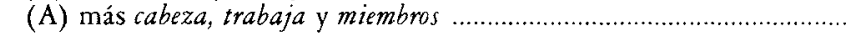 & 714 & 746 \\
\hline E) & 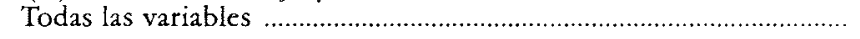 & 837 & 786 \\
\hline \multicolumn{4}{|c|}{ Reducción en la chi-cuadrada del modelo: } \\
\hline & De E a D (contribución de educación y universidad) & $123^{*}$ & $40^{*}$ \\
\hline & De D a C (contribución de miembros) & $60^{*}$ & $71^{*}$ \\
\hline & 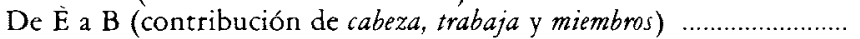 & $113^{*}$ & $64^{*}$ \\
\hline
\end{tabular}

* Significativo al nivel de 0,001 .

Todos los modelos incluyen a lambda como variable independiente de control.

importantes para comprender las pautas de desigualdad entre las mujeres que entre los varones.

Veamos ahora cuáles son los efectos concretos que ejercen cada una de las variables sobre la probabilidad de haber acudido a dentista privado. En la tabla 4 se presentan los parámetros estimados para el modelo que incluye todas nuestras variables (el modelo $\mathrm{E}$ de la tabla 3 ). Para mujeres y varones, los recursos familiares (renta, prestigio) aumentan la probabilidad de haber acudido a dentista privado. Residir en la provincia de Barcelona, o cerca de la frontera francesa, también aumenta la probabilidad, tal y como habíamos predicho. Características individuales tales como la educación y el trabajar fuera del hogar también aumentan la probabilidad de mujeres y varones. El número de miembros de la unidad familiar ejerce también el efecto negativo esperado. Urbanización y ser cabeza de familia solamente ejercen efectos significativos en el caso de los varones y, en contra de lo esperable, ambas variables tienden a reducir la probabilidad de haber acudido a dentista privado. La escasa significatividad de la variable cabeza sugiere que los beneficios derivados de ejercer la autoridad de decisión en el seno de la familia quizá estén más determinados por estar empleado fuera del hogar. El haber acudido a la universidad solamente es significativo en el caso de las mujeres. El signo negativo sugiere que el efecto positivo de la variable de años de educación se atenúa para valores superiores a quince años de educación formal.

Nótese, por último, que en este modelo E para mujeres y varones la variable lambda es significativa. Esto quiere decir que el sesgo de selección 


\section{TABLA 4}

Parámetros de máxima verosimilitud estimados para el modelo logit midiendo la probabilidad de que la persona entrevistada visitara un dentista privado la última vez que fue al dentista

\begin{tabular}{|c|c|c|c|c|c|c|}
\hline \multirow{2}{*}{$\begin{array}{c}\text { Variables } \\
\text { independientes }\end{array}$} & \multicolumn{3}{|c|}{ Mujeres } & \multicolumn{3}{|c|}{ Varones } \\
\hline & (E) & $(F)$ & (G) & (E) & $(F)$ & (G) \\
\hline Renta ..... & $0,634(3)$ & $0,568(2)$ & $0,641(3)$ & $0,628(3)$ & $-0,505$ & $0,585(3)$ \\
\hline Prestigio ...... & $0,014(3)$ & $0,014(3)$ & $0,011(3)$ & $0,013(3)$ & $0,015(3)$ & 0,005 \\
\hline 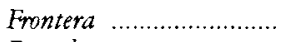 & $0,239(2)$ & $0,246(2)$ & $0,245(2)$ & $0,180(1)$ & $0,200(1)$ & $0,187(1)$ \\
\hline Barcelona & $0,206(2)$ & $0,205(2)$ & $0,204(2)$ & $0,299(3)$ & $0,312(3)$ & $0,297(3)$ \\
\hline Urbanización ................... & 0,038 & 0,039 & 0,040 & $-0,105(3)$ & $-0,093(3)$ & $-0,101(3)$ \\
\hline Educación & $0,082(3)$ & $0,069(2)$ & $0,062(2)$ & $0,047(3)$ & $-0,081(2)$ & 0,002 \\
\hline Universidad & $-0,346(1)$ & $-0,246$ & $-0,324$ & 0,107 & $1,395(3)$ & 0,542 \\
\hline Trabaja ........................ & $0,366(3)$ & $0,535(2)$ & $0,560(3)$ & $0,145(2)$ & $0,411(1)$ & 0,071 \\
\hline Cabeza. & 0,109 & $-0,331$ & $-0,602(1)$ & $-0,192(1)$ & $-0,153$ & $-0,228$ \\
\hline Miembros ......................... & $-0,117(3)$ & $-0,118(3)$ & $-0,119(3)$ & $-0,117(3)$ & $-0,113(3)$ & $-0,117(3)$ \\
\hline \multicolumn{7}{|l|}{ INTERACCIONES } \\
\hline \multicolumn{7}{|l|}{ Renta con: } \\
\hline Universidad ............... & 一 & $-0,105$ & - & - & $-1,373(3)$ & - \\
\hline Educación & - & 0,013 & - & -_. & $0,153(3)$ & - \\
\hline Trabaja & - & $-0,212$ & - & - & $-0,170$ & - \\
\hline 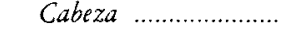 & - & $0,730(1)$ & - & - & $-0,045$ & - \\
\hline \multicolumn{7}{|l|}{ Prestigio con: } \\
\hline Universidad ................. & - & - & $-1,190$ & - & - & $-11,539$ \\
\hline Educación .................... & - & - & 0,523 & - & - & $1,248(1)$ \\
\hline Trabaja ........................ & - & - & 5,136 & - & - & 2,399 \\
\hline Cabeza ..................... & - & - & $19,929(2)$ & - & - & 0,899 \\
\hline Lambda & $0,102(1)$ & $0,097(1)$ & $0,097(1)$ & $0,220(3)$ & $0,231(3)$ & $0,214(3)$ \\
\hline Constante .......................... & $-0,547(3)$ & $-0,477(2)$ & $-0,429(2)$ & $-0,610(3)$ & 0,106 & $-0,269$ \\
\hline \multicolumn{7}{|l|}{ Modelo: } \\
\hline 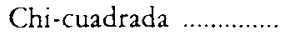 & 836,91 & 842,56 & 846,90 & 786,39 & 821,10 & 792,10 \\
\hline $\mathrm{R}$ & 0,253 & 0,253 & 0,254 & 0,250 & 2,255 & 0,250 \\
\hline Grados de libertad & 10627 & 10623 & 10623 & 9644 & 9640 & 9640 \\
\hline
\end{tabular}

(1) $\mathrm{p}<0,05$.

(2) $\mathrm{p}<0,01$.

(3) $\mathrm{p}<0,001$.

Todas las observaciones han sido ponderadas por el coeficiente provincial respectivo. Los parámetros estimados para renta, urbanización y todos los términos de interacción aparecen multiplicados por 1.000 . 
en la muestra es sistemático. Omitir la variable lambda produciría una situación en la que los términos de error estarían correlacionados con una variable importante no incluida en el modelo $y$, por tanto, todas nuestras estimaciones estarían sesgadas. El signo positivo de lambda también era esperable, puesto que el hecho de que una persona esté en la submuestra de las que han acudido al dentista (público o privado) al menos una vez en el pasado puede suponer una mayor preocupación con la salud dental que incline a acudir al dentista privado.

Las columnas F y $\mathrm{G}$ en la tabla 4 presentan modelos en los que se han añadido términos de interacción, esto es, variables que son el producto de la multiplicación de otras dos variables incluidas ya en el modelo. Los términos de interacción de las variables de educación (educación y universidad) con la renta familiar y el prestigio ocupativo del cabeza de familia son significativos para los varones, pero no para las mujeres. Por tanto, los varones sacan más partido de su educación a medida que aumentan los recursos familiares totales. Este efecto queda atenuado a partir del decimoquinto año de educación debido al signo negativo de la interacción de la renta con universidad. Un resultado muy interesante es que las mujeres que son cabeza de familia sacan partido de tal condición solamente cuando a medida que aumentan la renta o el prestigio ocupativo del cabeza de familia (es decir, ellas mismas). Estos extremos pueden constatarse al comprobar en los modelos $\mathrm{F}$ y $\mathrm{G}$ para mujeres que el parámetro de cabeza no es significativo $o$, en el caso del modelo $G$, es negativo. Sin embargo, los términos de interacción son significativos y positivos. Dejemos constancia, por último, que los modelos F y $G$ para mujeres mejoran la capacidad explicativa del modelo $\mathrm{E}$ con un nivel de significación del 5 por 100. En el caso de los varones el nivel de significación es notablemente mejor, del 0,1 por 100 .

\section{CONCLUSIONES}

Los resultados empíricos sobre la desigualdad de acceso a los cuidados privados de salud dental respaldan la mayoría de las hipótesis sobre las pautas inter e intrafamiliares de estratificación social, a excepción de los efectos de la urbanización y del hecho de ser cabeza de familia. En conjunto, las características personales y la posición de la persona en el seno de la familia aumentan significativamente el poder explicativo del modelo básico que incluye, solamente, las variables de recursos familiares y de localización geográfica. Tal y como era esperable, tanto la renta familiar como el estatus ocupativo del cabeza de familia aumentan la probabilidad de acudir a dentista privado. El resultado de que el ser cabeza de familia no aumenta significativamente la probabilidad de haber visitado un dentista privado sugiere que existen otros factores, quizá el trabajar fuera del hogar, que explican 
completamente la dinámica de autoridad y poder dentro de la familia. Otra interpretación "es, por supuesto, que existe un componente de conducta altruista que incita a las personas que son cabeza de familia a no sacar partido de tal condición. También se ha descubierto empíricamente que la proximidad geográfica a cuidados dentales más baratos (y quizá también de mejor calidad) al otro lado de la frontera francesa aumenta la probabilidad de consulta privada. Dado que la población española presenta niveles de salud dental comparativamente bajos, incluso entre las generaciones más jóvenes, y de que existen desigualdades pronunciadas a la hora de poder acudir a dentistas privados, pueden formularse dos consejos generales de política sanitaria. En primer lugar, el precio de los servicios dentales privados determina, en parte, el consumo de tales servicios por parte de la población, tal y como se deduce de los efectos sistemáticos y consistentes de la variable de proximidad a la frontera francesa. En segundo lugar, dados los recursos familiares totales, las personas con menos recursos personales (las niñas, niños y las mujeres que no trabajan fuera del hogar) son las que salen perdiendo a la hora de repartir los recursos que las familias destinan a cuidados de salud dental.

\section{BIBLIOGRAFIA}

ACKER, J. (1973): "Women and social stratification: A case of intellectual sexism", American Journal of Sociology, 78 (4): 936-945.

ALDRICH, John H., y NELSON, Forrest D. (1984): Linear Probability, Logit, and Probit Models, Beverly Hills, California: Sage.

ALLEN, S. (1982): "Gender inequality and class formation", en Anthony Giddens y Gavin MacKenzie (eds.), Social Class and the Division of Labour: Essays in Honour of Ilya Neustadt, Cambridge: Cambridge University Press, pp. 137-147.

ARnLjor, H. A., et al.( eds.) (1985): Oral Health Care Systems: An International Collaborative Study, Londres: Quintessence.

BARON, J. N. (1984): "Organizational perspectives on stratification", Annual Review of Socio$\log y, 10: 37-69$.

BERK, R. A. (1983): "An introduction to sample selection bias in sociological data", American Sociological Review, 48 (3): 386-398.

BIELBY, W. T., y BARON, J. N. (1986): "Men and women at work: Sex segregation and statistical discrimination", American Joumal of Sociology, 91: 759-799.

Blau, Peter M., y DUNCAN, Otis Dudley (1967): The American Occupational Structure, Nueva York: John Wiley.

BLOSSFIELD, H.-P. (1987): "Labor-market entry and the sexual segregation of careers in the Federal Republic of Germany", American Joumal of Sociology, 93: 89-118.

COHEN, L. K.; BAILIT, H. L., y BARMES, D. E. (1987): "International Collaborative Study of Oral Health Outcomes", International Sociology, 2 (4): 419-426.

Davis, P. (1981): "Culture, inequality, and the pattern of dental care in New Zealand", Social Science and Medicine, 1SA: 801-805.

De Miguel, Jesús M. (1985): La salud pública del futuro, Barcelona: Ariel.

- (1986): "Health sector structures: The case of Spain", Social Science and Medicine, 22 (2): $233-246$. 
DelPHY, C. (1979): "Sharing the Same Table: Consumption and the Family", en Chris Harris (ed.), The Sociology of the Family: New Directions for Britain, Sociological Review Monograph No. 28, Keele, Inglaterra: University of Keele, pp. 214-231.

EDGELL, Stephen (1980): Middle-Class Couples: A Study of Segregation. Domination and Inequality in Marriage, Londres: George Allen \& Unwin.

Galbraith, John Kennet (1973): Economics and the Public Purpose, Boston, Massachusetts: Houghton, Mifflin.

GILLESPIE, D. L. (1971): "Who has the power? The marital struggle", Joumal of Marriage and the Family, 33 (3): 445.458.

GOVE, W. R., y HuGHES, M. (1979): "Possible causes of the apparent sex differences in physical health: An empirical investigation", American Sociological Review, 44 (1): 126146.

HANUSheK, Eric A., y JaCKSON, John E. (1977): Statistical Metbods for Social Scientists, Orlando, Florida: Academic Press.

HARRELL, Frank E. (1986): "The Logist Procedure", en SAS, Supplemental Library User's Guide. Version 5 Edition, Cary, Carolina del Norte: SAS Institute, pp. 269-293.

HECHTER, Michael (1987): Principles of Group Solidarity, Berkeley, California: University of California Press.

HECKMAN, J. J. (1979): "Sample selection bias as a specification error", Econometrica, 47 (1): 153-161.

HOMER, M.; LeONARD, A., y TAYLOR, P. (1985): "The burden of dependency", Sociological Review Monograph, 31: 77-92.

HufF, Darrell [1954] (1982): How to Lie with Statistics, 36.2 ed., Nueva York: W. W. Norton.

INGLE, J. I., y BLAIR, P. (eds.) (1978): Intemational Dental Care Delivery Systems: Issues in Dental Health Policies, Cambridge: Ballinger.

JENKINS, R. M. (1987): "Gender differentials in earnings determination in the contemporary Hungarian labor force", artículo presentado en la Reunión Anual de la Population Association of America, Chicago, Illinois, 30 de abril.

Kalleberg, A. L., y Sørensen, Aage B. (1979): "The sociology of labor markets", Annual Review of Sociology, 5: 351-379.

KANTER, Rosabeth Moss (1977): Men and Women of the Corporation, Nueva York: Basic.

Kelsall, Keith; Kelsall, Helen M., y ChISHOlm, Lynne [1974] (1984): Stratification: An Essay on Class and Inequality, 2.2 ed., Londres: Longman.

LAND, H. (1977): "Inequalities in Large Families: More of the Same or Different?", en Robert Chester y John Peel (eds.), Equalities and Inequalities in Family Life, Londres: Academic Press, pp. 163-176.

LIPSET, Seymour Martin, y BENDIX, Reinhard [1959] (1967): Social Mobility in Industrial Society, Berkeley, California: University of California Press.

OECD (1985): Measuring Health Care, 1960-1983: Expenditure, Costs, and Performance, París: OECD.

Oral Health UNIT (1980): International Collaborative Study of Dental Manpower Systems: Interim Report, Ginebra: World Health Organization.

OrEN, L. (1973): "The welfare of women in laboring families: England, 1860-1950", Feminist Studies, 1 (3-4): 107-125.

ORZACK, Louis H. (1981): "New profession by fiat: Italian dentistry and the European Common Market", Social Science and Medicine, 15A: 807-816.

PAHL, J. (1980): "Patterns of money management within marriage", Joumal of Social Policy, 9 (3): $313-335$.

PARKIN, Frank (1971): Class Inequality and Political Order: Social Stratification in Capitalist and Communist Societies, Nueva York: Praeger.

- (1978): "Social Stratification", en Tom Bottomore y Robert Nisbet (eds.), A Histori of Sociological Analysis, Nueva York: Basic, pp. 599-632.

ROSENZWEIG, M. R. (1986): "Program interventions, intrahousehold distribution and the welfare of individuals: Modeling household behavior", World Development, 14 (2): 233 243 . 
SAFILIOS-ROTHSCHILD, C. (1970): "The study of family power structure: A review 19601969", Joumal of Marriage and the Family, 32 (4): 539-552.

SHRYOCK, Henry S., et al. (1980): The Methods and Materials of Demography, cuarta impresión (revisada), vol. 1, Washington, D. C.: Government Printing Office.

STACEY, Margaret (1969): Comparability in Social Research, Lodres: Heinemann.

SzINOVACZ, M. E. (1977): "Role allocation, family structure and female employment", Journal of Marriage and the Family, 39 (4): 781-791.

Treiman, Donald J. (1977): "Appendix A: Standard International Occupational Prestige Scale", en Occupational Prestige in Comparative Perspective, Nueva York: Academic Press, Pp. 235-260.

Treiman, D. J., y RoOS, P. A. (1983): "Sex and earnings in industrial society: A nine-nation comparison", American Joumal of Sociology, 89: 612-650.

VeBLEN, Thorstein [1899] (1979): The Theory of the Leisure. Class, Nueva York: Penguin. Traducción al castellano en Madrid: Hyspamérica, 1987.

WEBER, Max [1922] (1956): Wirtschaft und Gesellschaft: Grundiss der Verstehenden Soziologie, 2 vols., Tübingen: J. C. B. Mohr. Traducción al castellano en México: Fondo de Cultura Económica, 1979.

WestergaARD, John, y Resler, Henrietta (1975): Class in a Capitalist Society: A Study of Contemporary Britain, Nueva York: Basic.

Willis, R. J. (1987): "What have we learned from Economics of the Family?", American Economic Review, 77 (2): 68-81.

WORLD BANK, The (1988): World Development Report 1988, Nueva York: Oxford University Press for the World Bank.

WRIGHT, Erik Olin (1985): Classes, Londres: Verso.

YOUNG, M. (1952): "Distribution of income within the family", British Joumal of Sociology, 3 (4): 305-321.

YoUNG, Michael, y WILLmotT, Peter (1973): The Symmetrical Family, Nueva York: Pantheon. 